\title{
Detection and Correction of Stair Mode Across an Optical Phased Array
}

\author{
Jason Wyman \\ Air Force Institute of Technology \\ WPAFB OH, 45433
}

\author{
Milo W. Hyde \\ Air Force Institute of Technology \\ WPAFB OH, 45433
}

\begin{abstract}
A conceptual system capable of detecting the occurrence of stair mode across an optical array has been developed and studied. This conceptual system utilizes a single camera, referred to as the stair mode imager, to image the irradiance pattern produced at the target. The collected image is then cross-correlated with a database of theoretical stair mode images to estimate the amount of stair mode across the array. Previous studies have been limited to computer simulations and are expanded here into hardware-based experimentation. These experiments further study the effects of stair mode and are aimed at validating previous simulation findings and further characterizing the ability and constraints of the stair mode imager.
\end{abstract}

\section{TABLE OF Contents}

1 InTRODUCTION $\ldots . . \ldots \ldots \ldots \ldots \ldots \ldots \ldots \ldots \ldots \ldots$

2 STAIR MODE................................ 1

3 MATCHED Filter ........................ 3

4 Simulation Tests ...................... 4

5 EXPERIMENTAL TeStS ....................... 7

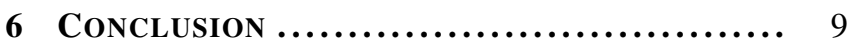

ACKNOWLEDGMENTS ....................... 9

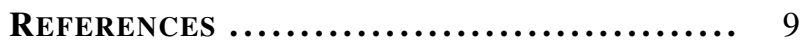

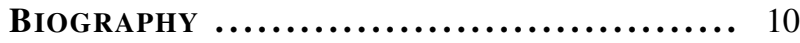

\section{INTRODUCTION}

Laser weapon research has been in part focused on reducing the size and weight of a weapon to make it well suited for use in aerial platforms. The U.S. Air Force's Airborne Laser program demonstrated directed energy's ability against a ballistic missile; however, the system was massive requiring structural modifications to a 747-400 freight aircraft in order to carry the megawatt class Chemical Oxygen Iodine Laser (COIL) as well as the supporting crew and equipment [1]. Focusing and steering of the high energy laser was accomplished through a $1.5 \mathrm{~m}$ mirror housed inside a swiveling turret located at the nose of the aircraft to reduce the negative effects of turbulent airflow across the turret [2]. While the system did successfully demonstrate a laser weapon's ability to detect, track, compensate for atmospheric disturbances, and use a high energy laser to cause critical structural failure to a representative ballistic missile in boosting phase [3], the size, weight, and complexity of the system made it impractical for use in smaller rapidly deployable aircraft systems.

The use of optical phased arrays allows for reduction in size and weight of a weapon system making them well suited for integration into airborne platforms. Through the use of an array of smaller and lighter fiber lasers coherently combined

${ }^{1}$ U.S. Government work not protected by U.S. copyright.

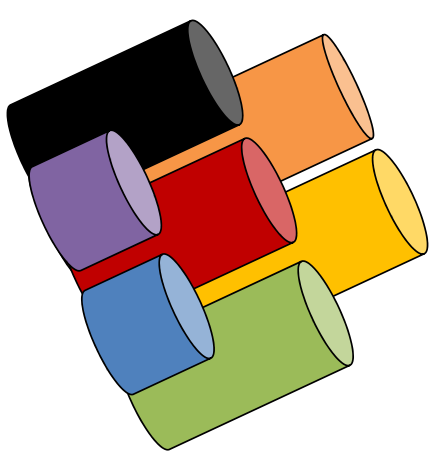

Figure 1: Depiction of stair mode on a seven element hexagonal array

at a target, the high power output required for a weapon system can be achieved [4]. However, the optical array introduces challenges, such as stair mode, not encountered using traditional monolithic beam-director systems. Stair mode is characterized as a stair-step pattern of piston phase across the optical array. This mode has the effect of steering the main array lobe away from the target point thereby shifting power into the array side lobes. This is an adverse condition for a laser weapon system where optimum performance is achieved when maximum power is delivered at a single point on the target. For this reason, the ability to accurately detect and correct for stair mode will be paramount in the development of an optical phased array laser weapon.

This paper studies an array comprised of seven circular elements arranged in a regular hexagon shape. The far-field irradiance patterns produced by the array are simulated with varying levels of piston phase applied to the array elements. These patterns are used in the construction of a matched filter that identifies the presence of stair mode in test patterns. The matched filter is tested using both simulation produced irradiance patterns as well as patterns produced using a hardwarebased experimental test bed. Finally the ability of the filter to be used in a closed-loop control algorithm to detect and remove stair mode from the piston phase of the array elements is demonstrated.

\section{STAIR Mode}

Stair mode is characterized as a stair-step pattern in piston phase across the phased array as depicted in Figure 1. For example, by incrementally adding $0.5 \lambda$ of piston to each column of elements in the array, an $x$ stair mode ( $x$ SM) is applied to the array as shown in Figure 2(a). Piston is added to each element in the array such that moving left to 


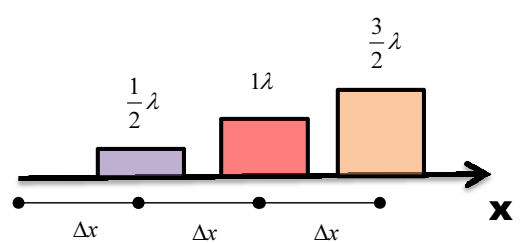

(a)

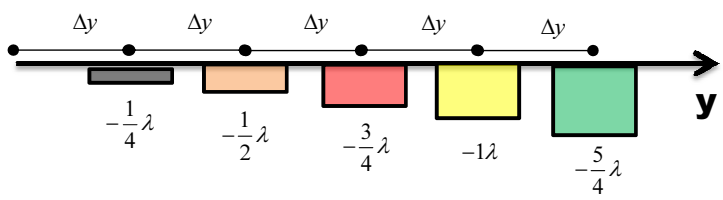

(b)

Figure 2: Depiction of applying an (a) $x$ SM of $0.5 \lambda$ and (b) $y \mathbf{S M}$ of $-0.25 \lambda$ to the seven element array

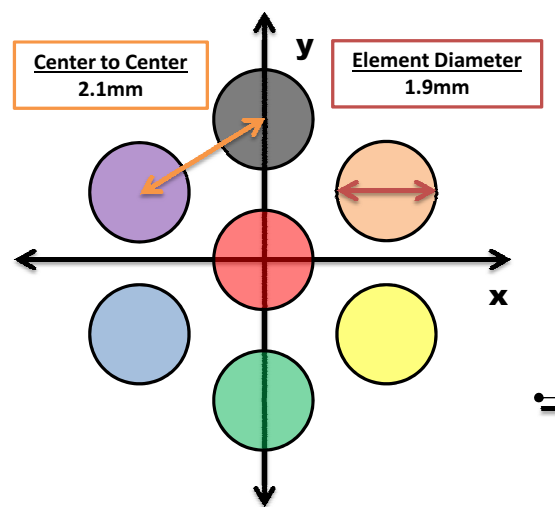

Figure 3: Seven element regular hexagon shaped array

right the first column of elements receives $0.5 \lambda$ of piston, the second column receives $2 \times 0.5 \lambda$, and third column receives $3 \times 0.5 \lambda$. This results in an effective slope of $0.5 \lambda / \Delta x$ across the three columns of elements, which is referred to here as $0.5 \lambda x$ SM. Similarly Figure 2(b) depicts $-0.25 \lambda y$ SM being applied to the array. Stair mode has the effect of steering the main array lobe away from the target point thereby shifting power into the array side lobes. This is demonstrated in Figure 4 using the far-field array patterns produced using the regular hexagon shaped array comprised of circular apertures depicted in Figure 3. The elements of the array each have a diameter of $1.9 \mathrm{~mm}$ with a center-to-center spacing between elements of $2.1 \mathrm{~mm}$. With zero stair mode across the array, Figure 4(a) shows that the central lobe of the far-field array pattern contains the vast majority of the power. When an $x \mathrm{SM}$ of $0.5 \lambda$ is applied to the array, the main lobe of the array is steered and power moves into the side lobes forming the two distinct lobes shown in Figure 4(b). If the intended target point in this scenario is the origin point of the figures, it is easy to see the negative effect of stair mode, as $0.5 \lambda$ of $x$ SM has caused the array to steer the majority of power to the left and right of the target point.

The shift of power into the side lobes is best seen through analysis of power in the bucket (PIB) as demonstrated by [5] and reproduced in Figure 5. The PIB is calculated by summing the irradiance contained in the region defined by the central spot of the far-field array pattern with zero stair mode applied to the array. The Figure clearly shows that as the value of stair mode moves from zero the PIB reduces to

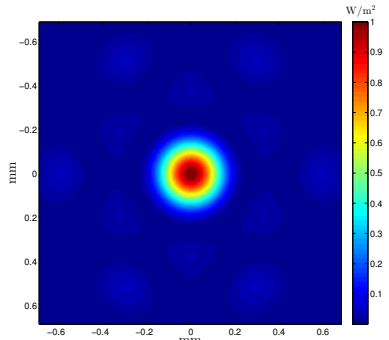

(a)

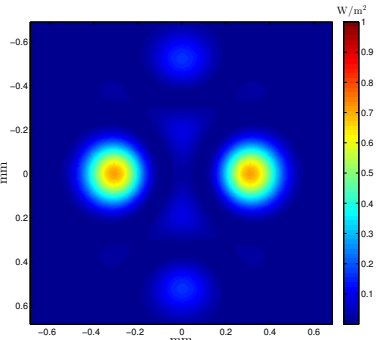

(b)
Figure 4: MATLAB rendering of the far-field irradiance patterns produced by seven element hexagonal array with (a) zero stair mode and (b) $0.5 \lambda x \mathrm{SM}$ across the array, patterns have been normalized using the on-axis irradiance value of the zero stair mode pattern

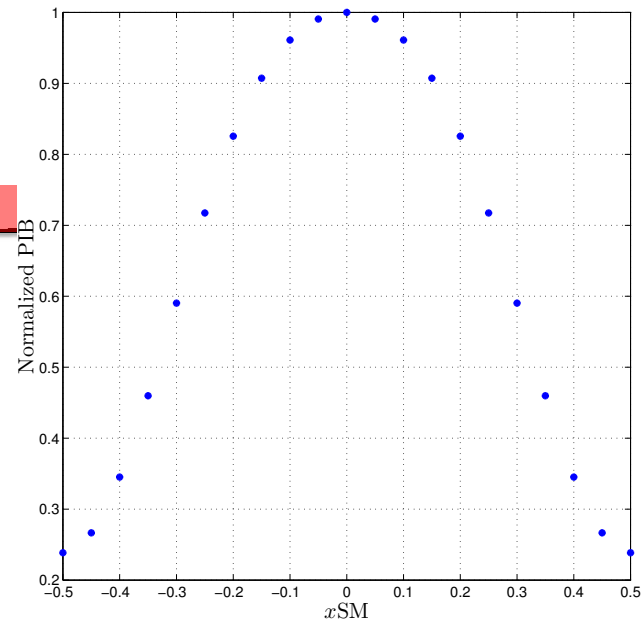

Figure 5: Normalized PIB for seven element hexagon shaped array produced with $0 \lambda y$ SM and varying $x$ SM applied to the array

a minimum value of $0.25 \%$ of the maximum PIB value when $0.5 \lambda x \mathrm{SM}$ is applied to the array. This is a significant drop in power at the target area and makes the detection and correction of stair mode critical for the successful development of this optical phased array weapon.

The transfer of power into the side lobes of the far-field pattern is due in part by $x$ SM being applied to the columns of array elements, but is principally due to the center-tocenter spacing between the array elements $d$ and the radiating wavelength $\lambda$. Reference [6] demonstrates that the far-zone electric field produced by an array of identical elements can be expressed as the product of the field produced by a single element of the array times the array factor for the array being evaluated. For a planer rectangular-shaped array, this array factor can be expressed as [6]

$$
A F(\theta, \phi)=\left[\frac{1}{M} \frac{\sin \left(\frac{M}{2} \varphi_{x}\right)}{\sin \left(\frac{\varphi_{x}}{2}\right)}\right]\left[\frac{1}{N} \frac{\sin \left(\frac{N}{2} \varphi_{y}\right)}{\sin \left(\frac{\varphi_{y}}{2}\right)}\right],
$$

where

$$
\begin{aligned}
& \varphi_{x}=k d_{x} \sin \theta \cos \phi+k \beta_{x}, \\
& \varphi_{y}=k d_{y} \sin \theta \sin \phi+k \beta_{y},
\end{aligned}
$$

$k=2 \pi / \lambda$ is the wavenumber, $d_{x}$ and $d_{y}$ are the centerto-center distances between array elements in the $x$ and 
$y$ directions respectively, and $\theta$ and $\phi$ denote the angular location of the far-field observation point. The remaining undefined symbols, $\beta_{x}$ and $\beta_{y}$, are the $x$ SM and $y$ SM phases used to steer the array.

The two $[\sin (A \psi) / A \sin (\psi)]$ functions in the array factor shown in Eq. (1) produce values of one at $\psi=n \pi$ where $n$ is an integer value. At these values the far-field array pattern may contain a local maximum/grating lobe [6]. If the main lobe of the pattern is said to be produced at a value of $\psi=0$, then the lobes that occur at $\psi=n \pi$ are considered grating lobes [6]. The irradiance of the grating lobes would be seen to increase or decrease as the value of $\psi$ moves closer to or away from $n \pi$.

When $\psi<2 \pi$, the array can be steered by values of $-\pi<$ $\beta<\pi$ without producing grating lobes - a feat achieved when $d_{x}, d_{y}<\lambda / 2$ [6]. At optical wavelengths, this can be difficult to achieve. For example, at $632 \mathrm{~nm}$ (wavelength used in the simulations and experiments here), the distance between elements could be no greater than $1.27 \mu \mathrm{m}-$ a distance much less than even the radius of a single element used in the array. Additionally, element sizes on the order of a micron would not be practical in an actual high-power operational weapon system, and so the ability to significantly steer (as is done for RF arrays) the far-field main lobe using the $\beta$ term without producing grating lobes is severely limited.

The relationship between the element spacing $d$ and wavelength $\lambda$ can be visually studied using the array factor for the seven element regular hexagon array [4]

$$
\begin{aligned}
\mathrm{AF}=1 & +2 \cos \left[2 \pi d\left(0.5 f_{x}-0.866 f_{y}\right)\right] \\
& +2 \cos \left[2 \pi d\left(0.5 f_{x}+0.866 f_{y}\right)\right]+2 \cos \left(2 \pi d f_{x}\right),
\end{aligned}
$$

where $f_{x}$ and $f_{y}$ are the related to the far-field $x$ and $y$ observation plane coordinates by $x / \lambda z$ and $y / \lambda z$, respectively. Rotating the array factor to match the array configuration used in this paper produces

$$
\begin{aligned}
\mathrm{AF}=1 & +2 \cos \left[2 \pi d\left(0.866 f_{x}-0.5 f_{y}\right)\right] \\
& +2 \cos \left[2 \pi d\left(0.866 f_{x}+0.5 f_{y}\right)\right]+2 \cos \left(2 \pi d f_{y}\right) .
\end{aligned}
$$

Plotting this array factor at varying sizes of $d$ with respect to $\lambda$ demonstrates that as $d$ becomes larger than $\lambda / 2$ the number of grating lobes in the array factor pattern increases. Multiplying the array factors depicted in Figure 6 by the irradiance pattern produced by the central element of the hexagonal array would produce the actual far-field pattern for the array. With this in mind, it is easy to see how the array factor, which is changed by the ratio of $d$ and $\lambda$ leads to side lobes.

\section{Stair Mode and Unobservable Modes}

The optimal performance of an optical phased array is achieved when the beams produced by the array elements are controlled such that they arrive at the target plane in phase allowing the beams to coherently combined producing the maximum irradiance point value for the array at the target [4]. As the beams from the seven element array travel to the target plane, they experience phase aberrations due to the telescopes/optics, atmosphere, and speckle aberrations that occur during the round trip path from the array to the target. Reference [7] demonstrates that if each element within the array acts as a receiver, there are sufficient measurements available to separate the speckle aberrations from the two aberration sources, telescope and atmospheric, that require correction to produce phasing of the beams at the target.

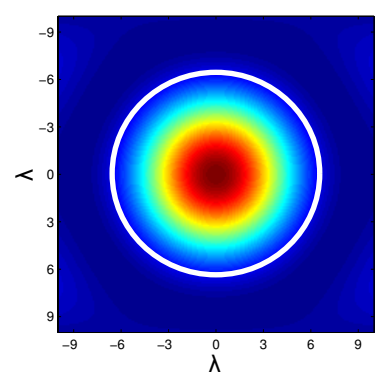

(a)

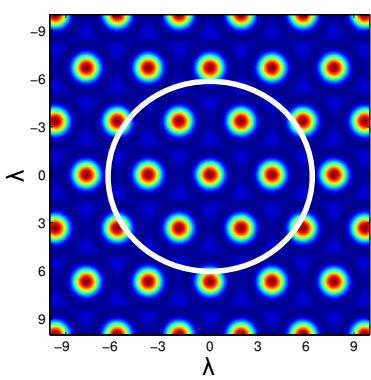

(b)

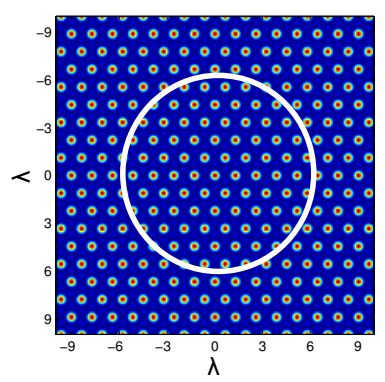

(c)

Figure 6: MATLAB rendering of the array factor for seven element hexagonal array with center-to-center distance between array elements of: (a) $\lambda / 2$ (b) $3 \lambda$ (c) $8 \lambda$. A unit value $\lambda$ and $z=10 \lambda$ are used to create the three patterns. The white circle depicts the far-field central

lobe for a single array element with diameter $2 \lambda$.

Using a least squares estimation, [7] demonstrates that with the measurements available for the seven element array there are set of unobservable modes that contain stair mode. Because stair mode can not be estimated using the measurements available and because of the impact it has on the power the array projects onto the target, an alternate method to detect and correct stair mode is required. The use of an imaging camera and matched filter has been proposed as a method to accomplish this. The camera is used to image the irradiance pattern produced at the target, this image is then correlated with a set of patterns with known stair mode values to identify the level of stair mode contained in the target image.

\section{MATChed Filter}

The expansion of matched filtering techniques used in onedimensional signal detection processes to two-dimensional object detection is well established [8], [9], [10]. In twodimensional space, the matched filter can be described as a spatial filter whose output can be used to determine the presence or absence of an object/image [9]. A spatial, or optical filter is matched to a signal $s(x, y)$ if the impulse response of the filter is

$$
h(x, y)=s^{*}(-x,-y)
$$

where * denotes the complex conjugate [8]. When an input signal $g(x, y)$ is applied to Eq. (6) the output $v(x, y)$ is

$$
\begin{aligned}
v(x, y) & =\int_{-\infty}^{\infty} \int_{-\infty}^{\infty} h(x-\xi, y-\eta) g(\xi, \eta) d \xi d \eta \\
& =\Im^{-1}\left[H\left(f_{X}, f_{Y}\right) G\left(f_{X}, f_{Y}\right)\right]
\end{aligned}
$$




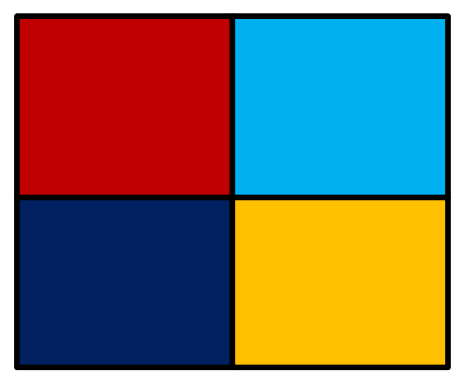

(a)

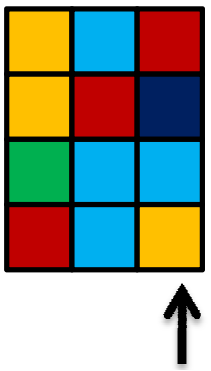

(b)
Figure 7: Depiction of (a) four pixel pattern (b) converted to a column of the $R$ filter

where $\Im^{-1}$ is the inverse Fourier transforms and $H$ and $G$ are the Fourier transforms of $h(x, y)$ and $g(x, y)$ respectively [8]. By substituting the Fourier transform of Eq. (6) into Eq. (7) it can be seen that when the input signal is equal to the desired signal the filter is matched to, i.e. $g(x, y)=$ $s(x, y)$, phase terms cancel [8] producing

$$
\begin{aligned}
v(x, y) & =\Im^{-1}\left[S\left(f_{X}, f_{Y}\right) S^{*}\left(f_{X}, f_{Y}\right)\right] . \\
& =\Im^{-1}\left|S\left(f_{X}, f_{Y}\right)\right|^{2} .
\end{aligned}
$$

An input not matched to the filter would not cancel the phase terms indicating the lack of a match [8].

Reference [9] expands matched filtering to a discrete image through vector correlation accomplished by calculation of the inner product

$$
f=\boldsymbol{m}^{T} \boldsymbol{f}_{u},
$$

where $\boldsymbol{m}$ is the matched filter vector and $\boldsymbol{f}_{u}$ is the image vector being analyzed. The resulting scalar value inner product, $f$, represents the degree of correlation between the filter vector and image vector.

Using the vector correlation method in Eq. (9), a filter matrix, referred to here as the $R$ filter, can be construction including both $x$ SM and $y$ SM far-field patterns.

\section{$R$ Filter Construction}

Construction of the $R$ filter is accomplished by simulating farfield patterns for the seven element array with known levels of $x \mathrm{SM}$ and $y \mathrm{SM}$ applied to the array. The patterns are converted to column vectors, normalized and stored in the filter matrix $R$ such that each column in the filter matrix represents an irradiance pattern with a known stair mode as shown in Figure 7. An irradiance pattern $i$ with an unknown value of stair mode is then converted to a column vector, normalized, and inner product calculated with the filter matrix $R$. This is the same calculation as shown in Eq. (9) but expressed now as

$$
\boldsymbol{S}=\boldsymbol{i}^{T} \boldsymbol{R}
$$

The results in matrix $S$ contain $N$ inner products, where $N$ corresponds to the number of patterns contained in the $R$ filter. The column in $\boldsymbol{S}$ that contains the largest inner product corresponds to the column in $R$, or the the theoretical array pattern that best matches the array pattern with the unknown stair mode value. Because of normalization of the image vectors, an inner product value of one would indicate a perfect match.

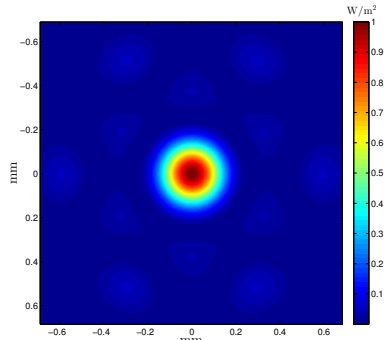

(a)

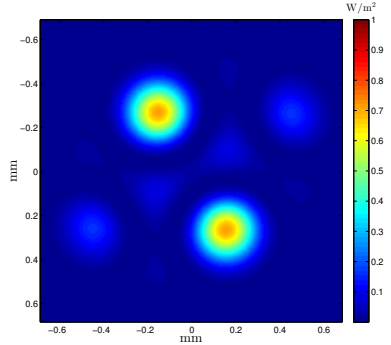

(c)

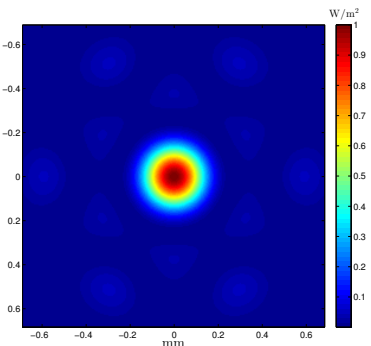

(b)

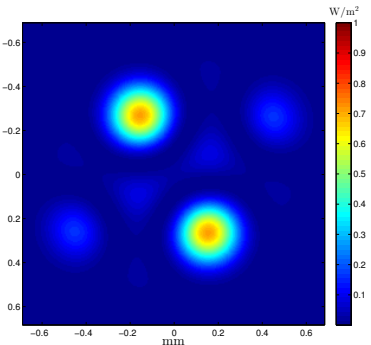

(d)
Figure 8: MATLAB rendering of duplicate far-field patterns contained in the $R$ filter, pattern produced with (a) $x \mathbf{S M = 0}|y \mathbf{S M = 0 , ( b ) ~} x \mathbf{S M = 0 . 5}| y \mathbf{S M = 0 . 5}$, (c) $x \mathbf{S M = 0 . 2 5} \mid y \mathbf{S M = 0 . 2 5}$, (d) $x \mathbf{S M = - 0 . 2 5} \mid y \mathbf{S M = - 0 . 2 5}$

The simulations and experiments conducted in this paper use an $R$ filter that is constructed using all combinations of $x \mathrm{SM}$ and $y \mathrm{SM}$ in incremental steps of $0.05 \lambda$ over the bounds of $-0.45 \lambda$ to $0.5 \lambda$. For example, an $x \mathrm{SM}$ of $-0.45 \lambda$ is applied to the array as well as a value of $y$ SM starting at $-0.45 \lambda$ up to and including $0.5 \lambda$ in increments of $0.05 \lambda$. The far-field pattern is simulated and stored in the $R$ filter at each $y$ SM increment. This process is continued for each $x$ SM value from $-0.45 \lambda$ up to and including $x$ SM $0.5 \lambda$ in increments of $0.05 \lambda$ producing a final $R$ filter containing 400 far-field patterns

\section{Simulation Tests}

The 400 pattern $R$ filter is tested using far-field array patterns produced by applying all combinations of $x \mathrm{SM}$ and $y \mathrm{SM}$ between $-0.45 \lambda$ and $0.5 \lambda$ in increments of $0.05 \lambda$. Initial tests of the $R$ filter appear to indicate that it is not always correctly identifying the amount of stair mode in the test images. Figure 8 shows two filter results where the filter incorrectly identifies the amount of stair mode. However, visual inspection of the images reveals that the patterns are identical and that the filter is not in error. Analysis of the difference between the input stair mode values and filter identified values reveals a difference of $0.5 \lambda$ in both $x \mathrm{SM}$ and $y \mathrm{SM}$ and is a result of duplicate patterns contained in the $R$ filter. Closer examination of the patterns contained in the filter reveals that there are only 200 distinct patterns in the filter and that duplicate patterns exist between array patterns separated by $\pm 0.5 \lambda x \mathrm{SM}$ and $y \mathrm{SM}$. The reason for the duplicate patterns is shown in Figure 9.

Stair mode is periodic over one wavelength. As a result, the array produced from adding Figure 9(a) to Figure 9(b) results in the array shown in Figure 9(c). After removing integer values of stair mode the resulting array has equal piston values applied to each element of the array, resulting 


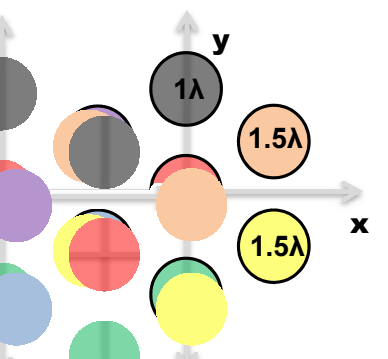

(a)

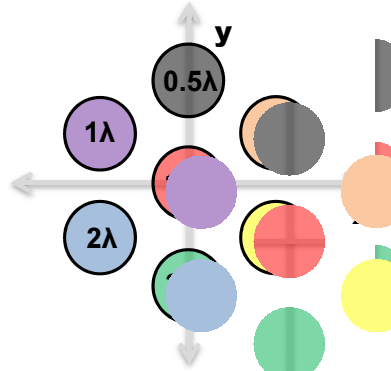

(b)

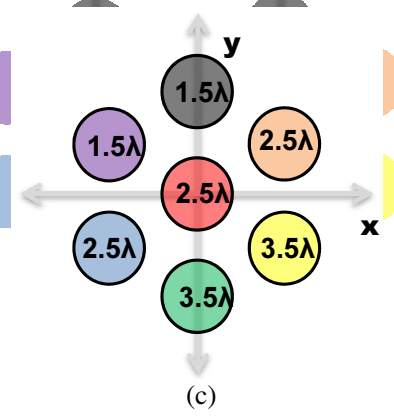

Figure 9: Demonstration of duplicate patters through adding arrays with (a) $0.5 x \mathrm{SM}$ and (b) $0.5 y \mathrm{SM}$ together producing and array (c) with $0.5 \lambda$ piston phase on each element resulting in zero stair mode across the array

in zero stair mode across the array. A similar analysis may be performed to demonstrate the other duplicate pairs.

A method to distinguish between the duplicate array patterns is not sought here because their occurrence does not hinder the primary reason for the filter, i.e, to detect and more importantly correct stair mode. The correction of stair mode is completed by determining the level of stair mode in the test pattern and subtracting the corresponding piston values from the individual element phase commands. Table 1 shows that regardless of whether the filter identifies the exact stair mode value or the duplicate pair, the resulting difference produced when subtracting the filter identified stair mode from the actual value of stair mode i.e, $x S M_{a}-x S M_{R} \mid y S M_{a}-y S M_{R}$, is either $0 \mid 0$ or $\pm 0.5 \mid \pm 0.5$ - all of which produce a farfield pattern that effectively has zero stair mode.

While a solution for duplicate patterns is not sought, their occurrence can be utilized to reduce the size of the $R$ filter. By plotting the $x \mathrm{SM}$ and $y \mathrm{SM}$ values, as in Figure 10 , the duplicate $x \mathrm{SM}$ and $y \mathrm{SM}$ values can be easily distinguished from one another. The blue central diamond in the figure contains the half of $x$ SM and $y$ SM values which yield nonduplicate patterns. By constructing a filter containing array patterns produced using just the stair mode values within the diamond region shown in Figure 10, the size of the filter is cut in half and now contains only 200 patterns. Additionally, the accuracy of the filter is further improved by adding a bestfit quadratic calculation. After the $S$ matrix is produced, the values adjacent to the maximum inner product are used to produce a best-fit quadratic that is then utilized to determine values of stair mode that exist between the $0.05 \lambda$ increments used to produce the filter.

The reduced filter with best-fit quadratic function is tested

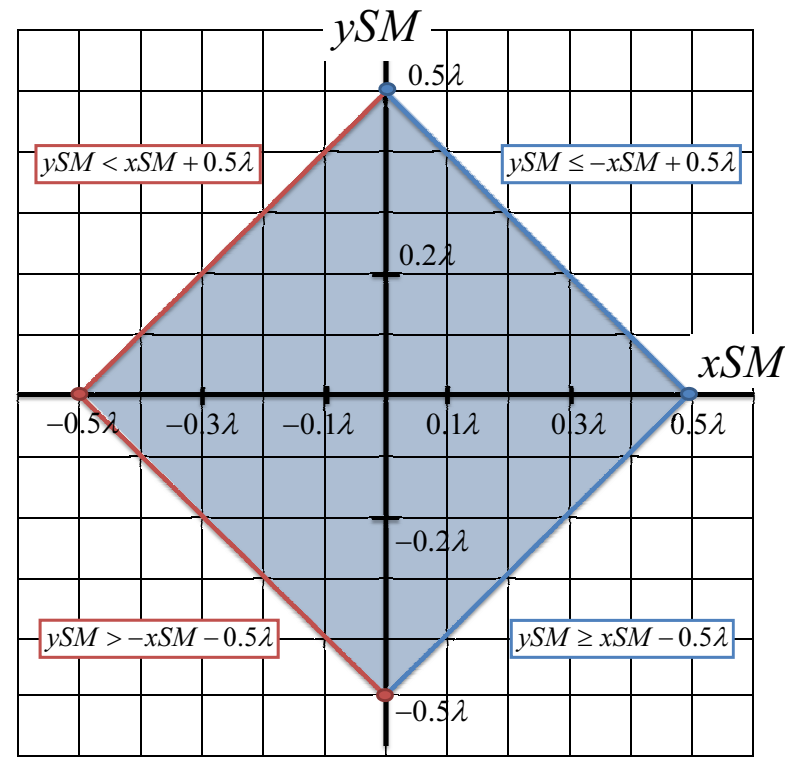

Figure 10: Depiction of region containing non-duplicate irradiance patterns (blue-shaded diamond), blue lines and dots are values included in the diamond and red lines and dots are values not included

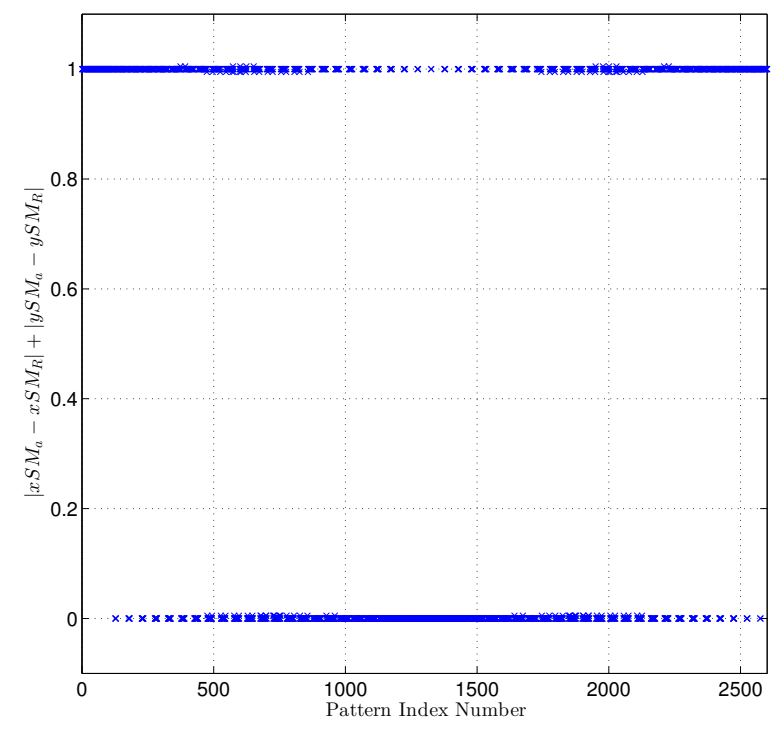

Figure 11: Results from $R$ filter simulation tests, value of zero or one indicates the filter has correctly identified the value of $x \mathbf{S M}$ and $y \mathbf{S M}$

using array patterns containing all combinations of $x$ SM and $y \mathrm{SM}$ between $-0.5 \lambda$ and $0.5 \lambda$ in increments of $0.02 \lambda$. This increment is selected as it produces at least two patterns between the increment value used in building the filter. Using the relationship shown in Table 1 , the filter identified stair mode values are subtracted from the actual stair mode values. The absolute values of the differences produced from the separate $x$ SM and $y$ SM are added together and the resulting sum will be either zero or one if a correct identification is made.

The results of the reduced $R$ filter tests are shown in Figure 11. It was found that in a few cases the filter was not exactly correct in identifying the stair mode value, however 
Table 1: Demonstration of Duplicate Pair Impact on Correction

\begin{tabular}{|c|c|c|c|c|c|c|c|}
\hline \multicolumn{2}{|c}{$S M_{a}$} & \multicolumn{2}{c|}{$S M_{R 1}$} & \multicolumn{2}{c|}{$S M_{R 2}$} & \multicolumn{2}{c|}{$S M_{a}-S M_{R}$} \\
\hline x-SM & $\mathbf{y - S M}$ & $\mathbf{x - S M}$ & $\mathbf{y - S M}$ & $\mathbf{x - S M}$ & $\mathbf{y - S M}$ & Diff 1 (x/y) & Diff 2 (x/y) \\
\hline 0 & 0 & 0 & 0 & 0.5 & 0.5 & $0 / 0$ & $-0.5 /-0.5$ \\
\hline 0.1 & 0.1 & 0.1 & 0.1 & -0.4 & -0.4 & $0 / 0$ & $0.5 / 0.5$ \\
\hline 0.3 & 0.3 & 0.3 & 0.3 & -0.2 & -0.2 & $0 / 0$ & $0.5 /-0.5$ \\
\hline-0.45 & -0.45 & -0.45 & 0.45 & 0.05 & -0.05 & $0 / 0$ & $-0.5 / 0.5$ \\
\hline-0.2 & -0.25 & -0.2 & -0.25 & 0.3 & 0.25 & $0 / 0$ & $-0.5 /-0.5$ \\
\hline
\end{tabular}

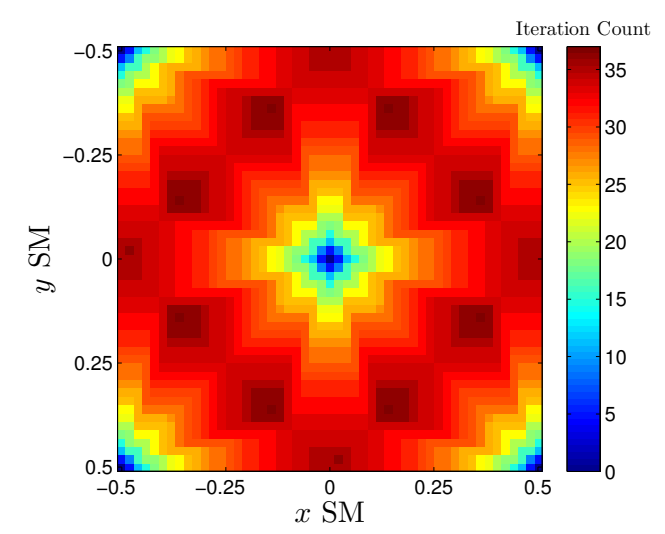

Figure 12: Plot of the number of iterations required to correct stair mode in simulation test

the error was found to be caused by an $x$ SM or $y$ SM value identification error of only $0.005 \lambda$ in each of the cases. An exact source of the error is not sought but is likely caused by a rounding error in the best fit calculation. This is based on additional tests of the filter where perfect results are achieved when using test patterns produced using the exact incremental value as used in making the filter and not using the the best fit quadratic.

\section{Simulation Closed-Loop Tests}

The $R$ filter is tested as part of a closed-loop correction system to demonstrate its ability to correct stair mode and produce a far-field pattern with zero stair mode. Closed-loop is performed using the following control law,

$$
c=1 a-0.1 b,
$$

where $a$ is the previous $x \mathrm{SM}$ or $y \mathrm{SM}$ array commands, $b$ is the filter identified residual SM value, and $c$ is the $x \mathrm{SM}$ or $y \mathrm{SM}$ array command. Correction is applied until

$$
\sqrt{\left(x S M_{R}\right)^{2}+\left(y S M_{R}\right)^{2}} \leq 0.01 \lambda
$$

where $x S M_{R}$ and $y S M_{R}$ are the filter identified values of the residual $x$ SM and $y$ SM respectively. The closedloop tests are performed using array patterns containing all combinations of $x \mathrm{SM}$ and $y \mathrm{SM}$ between $-0.5 \lambda$ and $0.5 \lambda$ in increments of $0.02 \lambda$. The number of iterations required to correct stair mode is shown in Figure 12. The mean number of iterations is found to be 31 with a maximum iteration of 37 and minimum value of zero iterations required to correct stair mode.

The five instances with zero iterations required to correct stair mode are shown in Table 2. As expected, the five values in
Table 2: Stair Mode Values with Minimum Correction Iterations

\begin{tabular}{|c|c|}
\hline$x$ SM $\mid y$ SM & Iteration Count \\
\hline \hline$-0.5 \mid-0.5$ & 2 \\
$-0.5 \mid 0.5$ & 2 \\
$0 \mid 0$ & 2 \\
$0.5 \mid-0.5$ & 2 \\
$0.5 \mid 0.5$ & 2 \\
\hline
\end{tabular}

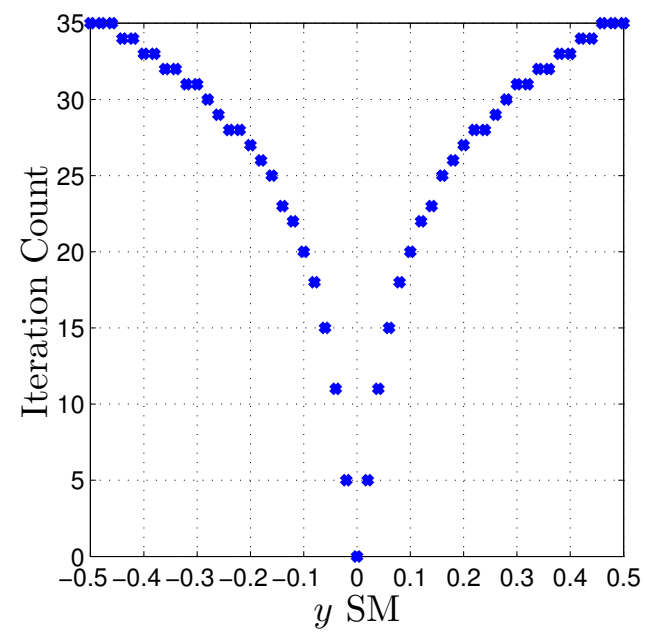

Figure 13: Plot of closed-loop simulation results with $x$ SM held constant at $0 \lambda$ with varying $y \mathbf{S M}$

the table are all stair mode values that effectively produce zero stair mode across the array. Figure 13 displays a plot of the iteration counts when $x \mathrm{SM}$ is zero and the $y \mathrm{SM}$ value varies between $-0.5 \lambda$ and $0.5 \lambda$ in increments of $0.02 \lambda$. The plot is as expected with the iteration count increasing as the $y \mathrm{SM}$ value moves farther away from zero.

Table 3 displays the eight $x$ SM and $y$ SM values that produced the maximum number of iterations to remove stair mode from the far-field array pattern. Figure 14 shows a plot of the iteration counts when the $x$ SM value is held at one of these values and the $y$ SM value is allowed to vary between $-0.5 \lambda$ and $0.5 \lambda$ in increments of $0.02 \lambda$. The figure contains two of the maximum iteration counts displayed in Table 3. It is surprising that the minimum iteration counts shown in the figure do not occur when $y$ SM approaches $0 \lambda$ as would have been expected from the results shown in Figure 13. The iteration count minimums instead occur at $x$ SM equal to $-0.36 \lambda$ and $y \mathrm{SM}$ equal to $\pm 0.5 \lambda-\mathrm{a}$ value 
Table 3: Stair Mode Values with Maximum Correction Iterations

\begin{tabular}{|c|c|}
\hline$x$ SM $\mid y$ SM & Iteration Count \\
\hline \hline$-0.36 \mid-0.14$ & 39 \\
$-0.36 \mid 0.14$ & 39 \\
$-0.14 \mid-0.36$ & 39 \\
$-0.14 \mid 0.36$ & 39 \\
$0.14 \mid-0.36$ & 39 \\
$0.14 \mid 0.36$ & 39 \\
$0.36 \mid-0.14$ & 39 \\
$0.36 \mid 0.14$ & 39 \\
\hline
\end{tabular}

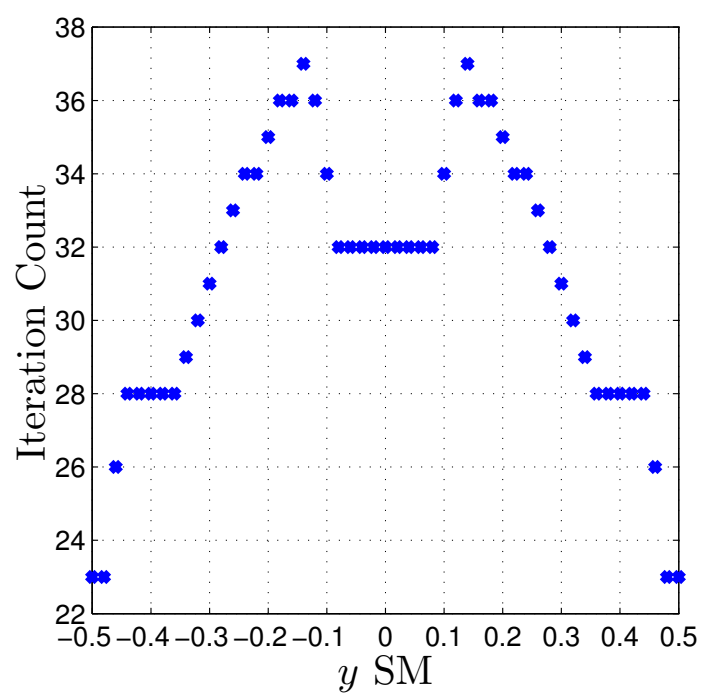

Figure 14: Plot of closed-loop simulation results with $x$ SM held constant at $\mathbf{- 0 . 3 6} \lambda$ and varying $y \mathbf{S M}$

that when plotted would seem to be farther from the origin than $x \mathrm{SM}=-0.36 \lambda$ and $y \mathrm{SM}=0 \lambda$. The reason for this can be explained by the existence of the duplicate far-field patterns and the reduced filter that utilizes just the SM values within the central diamond. The iteration count minimum exists outside the diamond region, thus when presented with the array patterns produced by these stair mode values, the filter identifies the stair mode value $x \mathrm{SM}=0.14 \lambda$ and $y \mathrm{SM}=0 \lambda$, a value that is closer to the origin.

\section{EXPERIMENTAL TESTS}

The experimental test bed shown in Figure 15, is used to produce far-field patterns at a target plane, image the patterns at the SMI plane, and use these images to test the $R$ filter. The actual optical table and hardware is shown in Figure 16. A $30 \mathrm{~mW} 632 \mathrm{~nm}$ Helium-Neon laser is used. Starting at the laser the orange dashed line in Figure 15 shows the beam path from the laser to the target. The beam expander immediately after the laser collimates and increases the size of the beam so that it overfills the face of the spacial light modulator (SLM). The iris immediately before the SLM controls the spatial extent of the light incident on the SLM allowing the light to be restricted to just the area being used to produce the seven element optical phased array. The $400 \mathrm{~mm}$ and $200 \mathrm{~mm}$ lenses image the face of the SLM at the $1 \mathrm{~m}$ lens

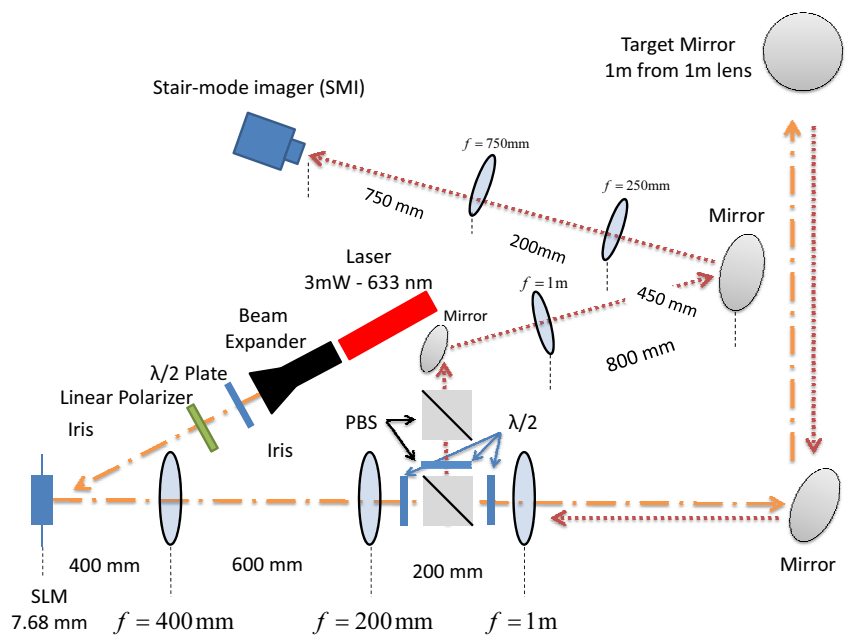

Figure 15: Schematic of experimental test bed

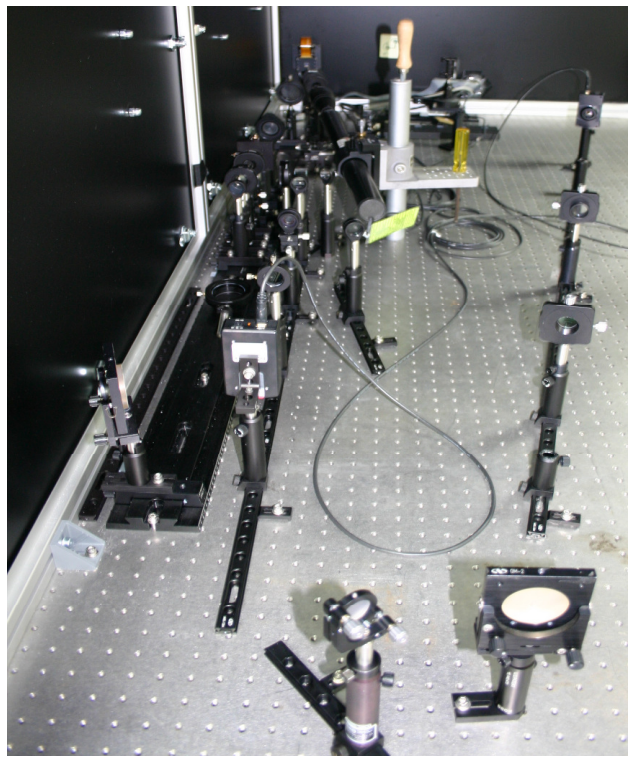

Figure 16: Photograph of experimental test bed

with a magnification of 0.5 . The $1 \mathrm{~m}$ lens is located at the image plane of the imaging system formed by the $400 \mathrm{~mm}$ and $200 \mathrm{~mm}$ lens; thus the far-field pattern of the array on the SLM, is produced at the focal plane of the $1 \mathrm{~m}$ lens [8]. The half waveplates around the $200 \mathrm{~mm}$ lens rotate the beam polarization and work in conjunction with the polarizing beam splitter cubes to send the maximum amount of outgoing light to the target plane as well as direct the maximum amount of light towards the SMI camera on the return path. On the leg return path (red-dashed arrows), the lens system formed by the $1 \mathrm{~m}, 1 \mathrm{~m}, 250 \mathrm{~mm}$ and $750 \mathrm{~mm}$ lenses image the irradiance pattern at the target in the SMI camera; this image is three times the size of the pattern at the target. Finally, the SMI is a Lumenera 125 (Lu125M) 1.3 Megapixel monochrome camera.

Sample images of the far-field pattern produced at the target plane and SMI plane are shown in Figure 17. The images presented in the figure are slightly saturated to better show the array pattern. Unsaturated images are used in the experiments to follow. The array produced in the SLM has the same 


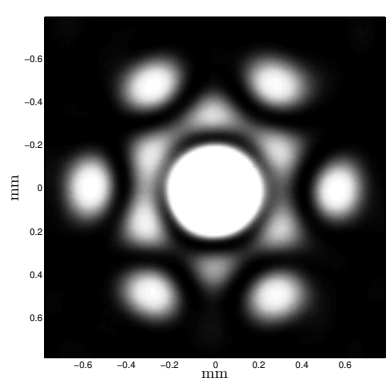

(a)

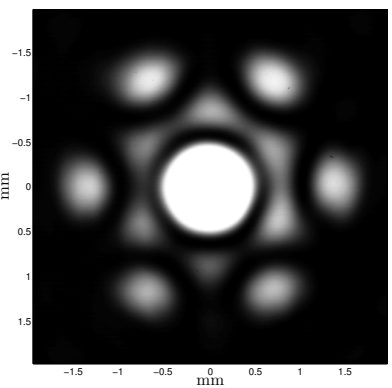

(b)
Figure 17: MATLAB rendering of the far-field pattern produced at the (a) target plane and (b) in the SMI camera

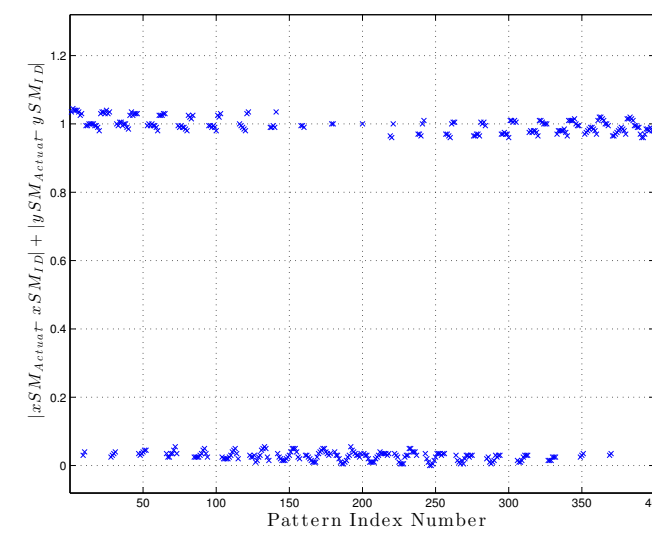

Figure 18: $R$ filter results obtained using far-field irradiance patters produced using the experimental test bed

shape and dimensions as the array used in simulations from Section 2.

\section{$R$ Filter Experimental Tests}

The $R$ filter is tested using the experimental setup described above by applying all combinations of $x$ SM and $y$ SM between $-0.45 \lambda$ and $0.5 \lambda$ in increments of $0.05 \lambda$, producing 400 test patterns. The test patterns are collected at the SMI camera plane. The $R$ filter results are shown in Figure 18. As previously performed, the filter identified $x$ SM and $y$ SM values are subtracted from the actual applied stair mode values and the absolute values of the two differences are added. The resulting sum will be zero or one if the filter correctly identified the level of $x \mathrm{SM}$ and $y \mathrm{SM}$.

The results shown in Figure 18 show that there are errors in identifying the value of stair mode in the patterns. The maximum, mean, and standard deviation of the errors are displayed in Table 4. A closer inspection of the irradiance patterns produced using the experimental test bed reveals the

Table 4: $R$ filter Experimental Test Errors

\begin{tabular}{|c|c|c|c|}
\hline & Max Error & Mean Error & Standard Deviation \\
\hline \hline$x$ SM & $0.03 \lambda$ & $0.0114 \lambda$ & $0.0113 \lambda$ \\
$y$ SM & $0.04 \lambda$ & $0.0165 \lambda$ & $0.0129 \lambda$ \\
\hline
\end{tabular}

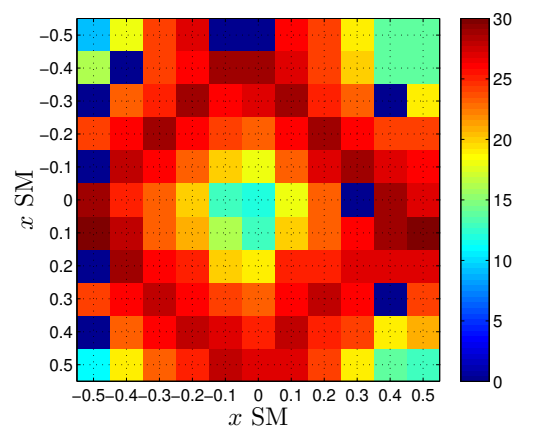

Figure 19: Plot of the number of iterations required to correct stair mode in experiment test

source of the errors to be an effective stair mode being added to the array by errors in the alignment of the experimental test bed.

\section{Closed-Loop Experimental Tests}

Using the closed-loop parameters from Eq. (11), a closedloop test is performed as in Section 4. The experimental closed-loop tests however require considerably more time to perform than the simulations. In an effort to reduce the data collection time the closed-loop experiment is performed using all combinations of $x \mathrm{SM}$ and $y \mathrm{SM}$ between $-0.5 \lambda$ and $0.5 \lambda$ in increments of $0.1 \lambda$ for the $x \mathrm{SM}$ and $y \mathrm{SM}$. The control law algorithm is the same as used in simulation. The correction is terminated when Eq. (12) is satisfied to within $0.025 \lambda$.

As in the simulation, the number of iterations required to correct stair mode is tracked and the results are shown in Figure 19. The mean number of iterations is found to be 22 with a maximum number of 30 and minimum number of zero iterations required to correct stair mode. The reduction seen in the mean and maximum iteration values is expected as the termination criterion is less stringent than in the simulation.

The relatively small number of data points displayed in Figure 19 makes a direct comparison to the simulation results in Figure 12 difficult. In an effort to alleviate this issue, the simulation results shown in Figure 13 and Figure 14 are experimentally reproduced by running the closed-loop experiment holding $x \mathrm{SM}=-0.36 \lambda$ and $x \mathrm{SM}=0 \lambda$ while varying $y \mathrm{SM}$ between $-0.5 \lambda$ and $0.5 \lambda$ in increments of $0.02 \lambda$. The plots of the iteration counts for both of these experiments are shown in Figure 20 and Figure 21, respectively. The results shown in Figures 20 and 21 show the characteristic shapes as displayed in the simulation results shown in Figures 14 and 13. The commanded values and residual stair mode values for one array pattern with $x \mathrm{SM}=-0.14 \lambda$ and y $\mathrm{SM}=-0.36 \lambda$ are presented in Figure 22. Additionally, the far-field array pattern collected in the SMI is shown at various correction iteration points in Figure 23. The iteration numbers shown have been selected to depict the correction path. As the control system removes stair mode from the far-field pattern, the vertex at the top most tip of the triangle formed by the three lobes in Figure 23(a) can be seen to move down and to the right towards the origin. As this lobe shifts closer to the origin it becomes brighter and the side lobes dimmer. Figure 24 shows the final pattern that results when the correction removes stair mode to within $0.025 \lambda$. 


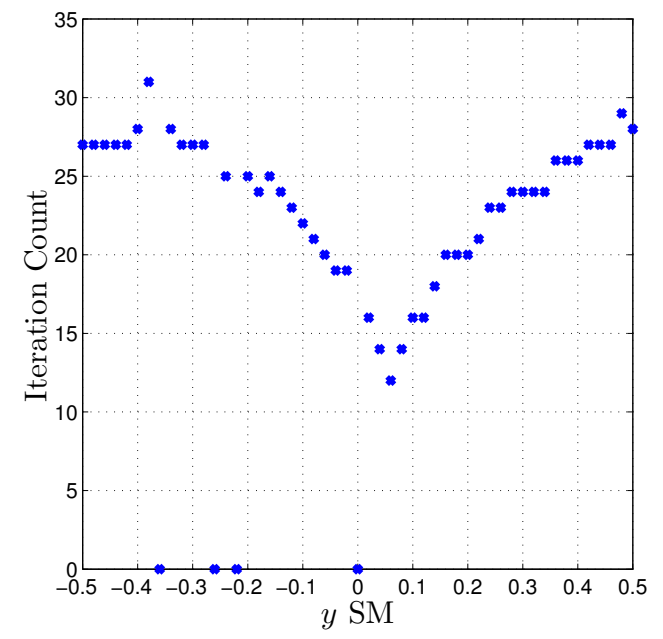

Figure 20: Plot of closed-loop experimental iteration counts to remove stair mode in array pattern generated with $x \mathbf{S M}=-0.36 \lambda$ and $y \mathbf{S M}$ between $-\mathbf{0 . 5} \lambda$ and $0.5 \lambda$ in increments of $0.02 \lambda$

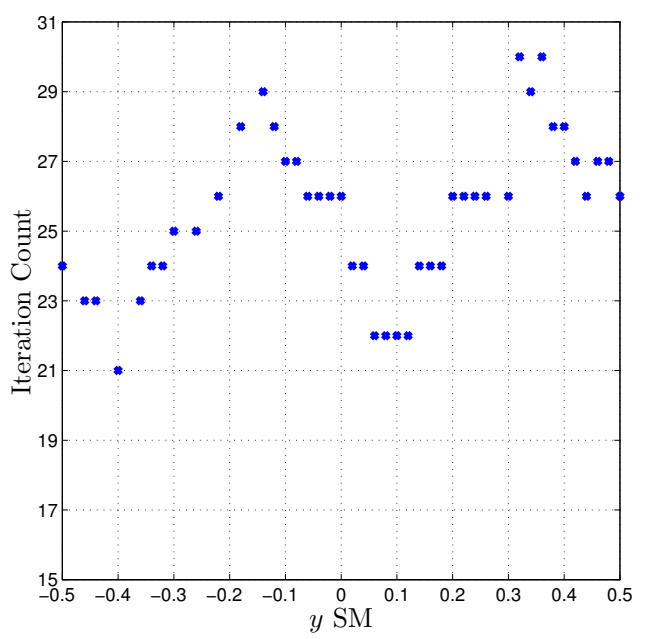

Figure 21: Plot of closed-loop experimental iteration counts to remove stair mode in array pattern generated with $x \mathbf{S M}=0.0 \lambda$ and $y \mathbf{S M}$ between $-0.5 \lambda$ and $0.5 \lambda$ in increments of $0.02 \lambda$

\section{Conclusion}

In this paper we have presented results of a potential matched filter approach for the detection and correction of stair mode across an optical phased array. Future work will entail more rigorous experimental testing of the filter, including effects of apodization.

\section{ACKNOWLEDGMENTS}

I would like to give a special thinks to fellow student $\mathrm{Mr}$. Franklin Jose, whose tireless efforts at calibrating the SLM and development of MATLAB algorithm to command the SLM were absolutely critical in the step-up of the experimental test bed. If not for his will not quit approach to improve upon the calibration, the results presented here would not have been possible.

An additional thanks is due to Mr. Mike Steinbock for

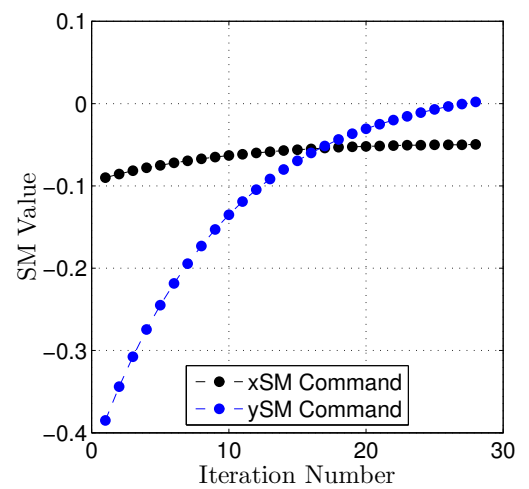

(a)

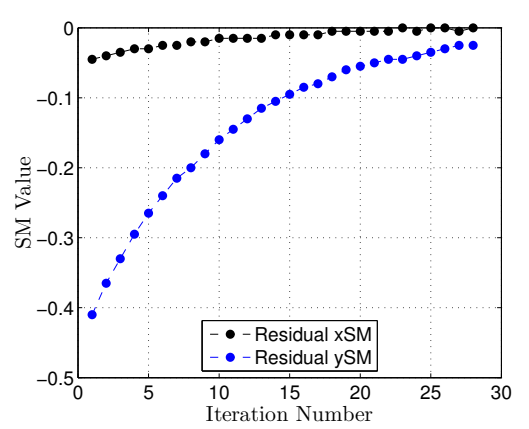

(b)

Figure 22: Plots of (a) array commands and (b) residual stair mode in the far-field pattern. Correction starts with a $x \mathbf{S M}=-0.14$ and $y \mathbf{S M}=-0.36$ image

the countless mentor hours he has provided in addition to troubleshooting advice that has helped me along the way.

Lastly a thank you is owed to The Optical Sciences Company (tOSC) for recommending the matched filter method for detection of stair mode.

\section{REFERENCES}

[1] S. E. Lamberson, "The airborne laser," Proc. SPIE, vol. 4760, pp. 25-33, 2002. [Online]. Available: http://dx.doi.org/10.1117/12.482116

[2] G. Forden, "The airborne laser," Spectrum, IEEE, vol. 34, no. 9, pp. 40-49, 1997.

[3] M. D. Agency, "The airborne laser test bed, fact sheet," http://www.mda.mil, January 2013.

[4] R. W. Berdine and A. R. Motes, Introduction to High Power Fiber Lasers. Albuquerque, NM: Directed Energy Professional Society, 2009.

[5] M. F. Spencer and M. W. Hyde, "An investigation of stair mode in optical phased arrays using tiled apertures," Proc. SPIE, vol. 8520, pp. 852 006-852 006-15, 2012. [Online]. Available: http://dx.doi.org/10.1117/12.942634

[6] C. A. Balanis, Antenna Theory, 3rd ed. Hoboken, NJ: John Wiley and Sons,Inc., 2005.

[7] G. A. Tyler, "Accommodation of speckle in objectbased phasing," J. Opt. Soc. Am. A, vol. 29, no. 5, pp. 722-733, May 2012. [Online]. Available: http://josaa.osa.org/abstract.cfm?URI=josaa-29-5-722 


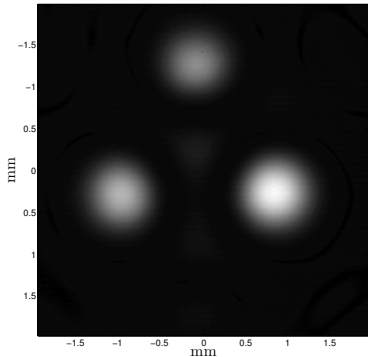

(a)

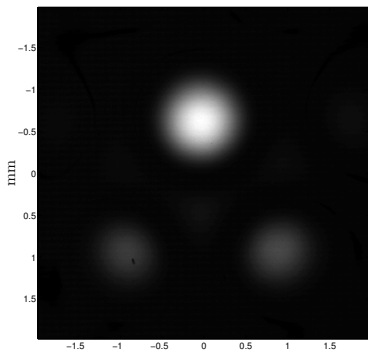

(c)

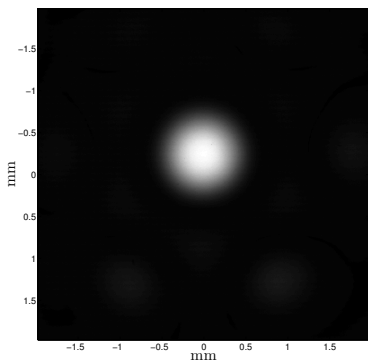

(e)

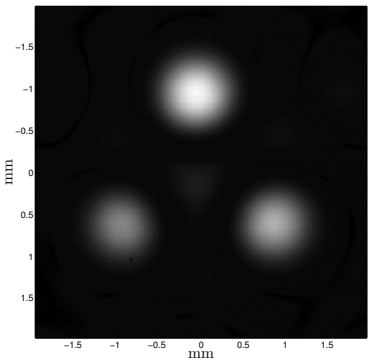

(b)

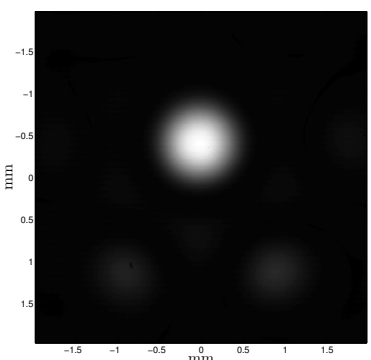

(d)

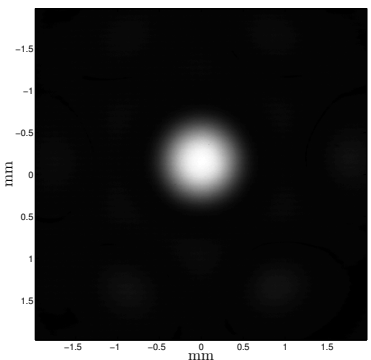

(f)
Figure 23: Far-field array pattern captured at iteration numbers: (a) 1, (b) 4, (c) 8, (d) 12, (e) 17, and (f) 21 . Correction starts with a $x \mathbf{S M}=-0.14$ and $y \mathbf{S M}=-0.36$ image

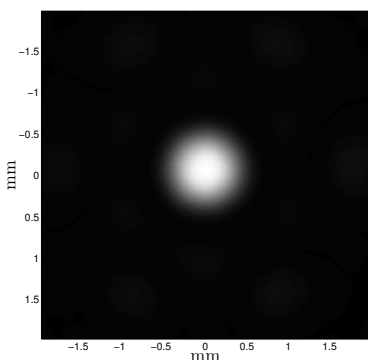

(a)

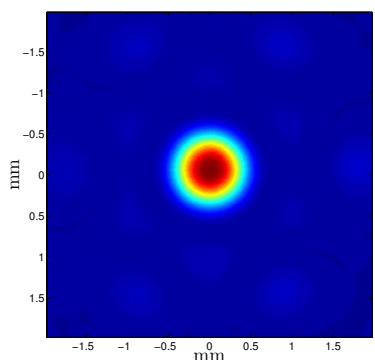

(b)
Figure 24: Far-field array pattern after completion of correction algorithm as seen in (a) the SMI camera and (b) MATLAB rendering of the image
[8] J. W. Goodman, Introduction to Fourier Optics, 3rd ed. Englewood, CO: Roberts and Company, 2005.

[9] W. K. Pratt, Digital Image Processing, 4th ed. Hoboken, NJ: John Wiley Sons, Inc., 2007.

[10] G. Turin, "An introduction to matched filters," Information Theory, IRE Transactions on, vol. 6, no. 3, pp. 311$329,1960$.

\section{BIOGRAPHY}

Jason Wyman received his B.S. degree in Electrical Engineering from the Illinois Institute of Technology in 2005 and an M.S. in Business Adminstration from Boston University in 2010. He commissioned as an officer in the U.S. Air Force in May of 2005 and has worked as project engineer on the Air Force's Theater Deployable Communications and Airborne Laser Communications Terminal programs as well as serving a tour in the Defense Contract Management Agency (DCMA) providing Government oversight at Northrop Grumman for the F-35 and Multi Platform Radar Technology Insertion Program (MP-RTIP). In December of 2008 he deployed to Iraq in support of a foreign military sales contract installing airfield lighting, navigation aids and Digital Airport Surveillance Radar (DASR) systems at Kirkuk airfield. He is currently working to complete his M.S. in Electrical Engineering at the Air Force Institute of Technology with an expected graduation date of March 2014. 